\title{
Online Vertex Exploration Problems in a Simple Polygon
}

\author{
Yuya HIGASHIKAWA ${ }^{\dagger \text { a) }}$, Nonmember and Naoki KATOH ${ }^{\dagger}$, Member
}

SUMMARY This paper considers online vertex exploration problems in a simple polygon where starting from a point in the inside of a simple polygon, a searcher is required to explore a simple polygon to visit all its vertices and finally return to the initial position as quickly as possible. The information of the polygon is given online. As the exploration proceeds, the searcher gains more information of the polygon. We give a 1.219-competitive algorithm for this problem. We also study the case of a rectilinear simple polygon, and give a 1.167-competitive algorithm.

key words: online algorithm, exploration, competitive analysis

\section{Introduction}

The Tohoku Earthquake attacked East Japan area on March 11,2011 . When such a big earthquake occurs in an urban area, it is predicted that many buildings and underground shopping areas will be heavily damaged, and it is seriously important to efficiently explore the inside of damaged areas in order to rescue human beings left there. With this motivation, this paper deals with Online Vertex Exploration Problems (OVEP for short) in a simple polygon. Given a simple polygon $P$, suppose the searcher is initially in the inside of $P$. Starting from the origin $o$, the aim of the searcher is to visit all vertices of $P$ at least once and to return to the origin as quickly as possible. The information of the polygon is given online. Namely, at the beginning, the searcher has only the information of a visible part of the polygon. As the exploration proceeds, the visible area changes. However, the information of the region which has once become visible is assumed to be accumulated. So, as the exploration proceeds, the searcher gains more information of the polygon, and determines which vertex to visit next based on the information obtained so far.

In general, the performance of an online algorithm is measured by a competitive ratio which is defined as follows. Let $\mathcal{S}$ denote a class of objects to be explored. When an online exploration algorithm ALG is used to explore an object $S \in \mathcal{S}$, let $|\mathrm{ALG}(S)|$ denote the tour length required to explore $S$ by ALG. Also, let $|\mathrm{OPT}(S)|$ denote the tour length required to explore $S$ by an offline optimal algorithm. Note that an algorithm is said to be offline optimal if it is optimal under the setting where all information of an object is given in advance. Then the competitive ratio of ALG is defined as

Manuscript received March 28, 2012.

Manuscript revised July 27, 2012.

†The authors are with the Department of Architecture and Architectural Engineering, Kyoto University, Kyoto-shi, 615-8540 Japan.

a) E-mail: as.higashikawa@archi.kyoto-u.ac.jp DOI: 10.1587/transinf.E96.D.489 follows.

$$
\sup _{S \in \mathcal{S}} \frac{|\operatorname{ALG}(S)|}{|\operatorname{OPT}(S)|} .
$$

Previous Work: OVEP has been extensively studied for the case of graphs. Kalyanasundaram et al. [10] presented a 16-competitive algorithm for planar undirected graphs. Megow et al. [8] recently extended this result to undirected graphs with genus $g$ and gave a $16(1+2 g)$-competitive algorithm. For the case of a cycle, Miyazaki et al. [9] gave an optimal 1.366-competitive algorithm. All these results are concerned with a single searcher. For the case of $p(>1)$ searchers, there are some results. Fraigniaud et al. [3] gave an $O(p / \log p)$-competitive algorithm for the case of a tree. Higashikawa et al. [6] gave $(p / \log p+o(1))$-competitive algorithm for this problem. Dynia et al. [2] showed a lower bound $\Omega(\log p / \log \log p)$ for any deterministic algorithm for the case of a tree.

There are some papers that are related to OVEP in geometric regions (see survey paper [5]). Kalyanasundaram et al. [10] studied the case of a polygon with holes where all edges are required to traverse. They gave a 17-competitive algorithm for this case. Hoffmann et al. [7] studied the problem that asks to find a tour in a simple polygon such that every vertex is visible from some point on the tour, and gave a 26.5-competitive algorithm.

Our Results: We will show a 1.219-competitive algorithm for OVEP in a simple polygon, and give a lower bound result that the competitive ratio is at least 1.040 within a certain framework of exploration algorithms. Also for rectilinear simple polygons, we give a 1.167-competitive algorithm, and a lower bound 1.034 .

\section{Fundamental Properties and the Algorithm Strategy}

In this paper, we define a simple polygon as a region in the plane (including the boundary) enclosed by a closed polygonal chain with no self-intersection. A closed polygonal chain is defined as an alternate sequence of vertices and edges $\left(v_{1}, e_{1}, v_{2}, e_{2}, \ldots, v_{n}, e_{n}\right)$ such that $e_{i}$ is a line segment connecting $v_{i}$ and $v_{i+1}$ for each $i(1 \leq i \leq n)$ where $v_{n+1}=v_{1}$ is assumed. A closed polygonal chain is said to have with no self-intersection if only consecutive (or the first and the last) edges intersect at their common endpoints. In the followings, we use the term a polygon to stand for a simple polygon.

Suppose that we are given a polygon $P$ and the origin 
$o$ in $P$. In what follows, we call a vertex of $P$ a polygon vertex, an edge of $P$ a polygon edge and the closed polygonal chain forming $P$ the boundary of $P$. Let $V=\left\{v_{1}, v_{2}, \ldots, v_{n}\right\}$ be a polygon vertex set sorted in clockwise order along the boundary of $P$ and $E=\left\{e_{1}, e_{2}, \ldots, e_{n}\right\}$ be a polygon edge set such that $e_{i}=\left(v_{i}, v_{i+1}\right)$ for $1 \leq i \leq n$ as above. For a polygon edge $e \in E$, let $v_{e}^{1}, v_{e}^{2}$ denote the endpoints of $e$ such that $v_{e}^{1}$ precedes $v_{e}^{2}$ in clockwise order, and let $|e|$ denote the length of $e$. Let $L$ denote the boundary length of $P$, namely $L=\sum_{e \in E}|e|$. For any two points $x, y \in P$, let $s p(x, y)$ denote the shortest path from $x$ to $y$ that lies in the inside of $P,|\operatorname{sp}(x, y)|$ be its length and $|x y|$ be the Euclidean distance from $x$ to $y$. Note that $s p(x, y)=s p(y, x)$ and $|x y| \leq|\operatorname{sp}(x, y)|$. Furthermore, for any two vertices $x, y \in V$, let $b p(x, y)$ denote the clockwise path along the boundary of $P$ from $x$ to $y$ and $|b p(x, y)|$ be its length.

For a point $x \in P$ and a polygon edge $e \in E$, let $T(x, e)$ denote the tour composed of paths $s p\left(x, v_{e}^{2}\right), b p\left(v_{e}^{2}, v_{e}^{1}\right)$ and $s p\left(v_{e}^{1}, x\right)$, and $|T(x, e)|$ be its length. Then, from $|T(x, e)|=$ $\left|s p\left(x, v_{e}^{1}\right)\right|+\left|s p\left(x, v_{e}^{2}\right)\right|+\left|b p\left(v_{e}^{2}, v_{e}^{1}\right)\right|$ and $\left|b p\left(v_{e}^{2}, v_{e}^{1}\right)\right|=L-$ $|e|,|T(x, e)|=L+\left|s p\left(x, v_{e}^{1}\right)\right|+\left|s p\left(x, v_{e}^{2}\right)\right|-|e|$ holds. The term $\left|s p\left(x, v_{e}^{1}\right)\right|+\left|s p\left(x, v_{e}^{2}\right)\right|-|e|$ represents the increase of the length from $L$, and thus we define

$$
\operatorname{inc}(x, e)=\left|\operatorname{sp}\left(x, v_{e}^{1}\right)\right|+\left|\operatorname{sp}\left(x, v_{e}^{2}\right)\right|-|e| .
$$

Note that $|T(x, e)|=L+\operatorname{inc}(x, e)$. Let $e_{\text {opt }} \in E$ be a polygon edge satisfying the following equation.

$$
\operatorname{inc}\left(o, e_{o p t}\right)=\min _{e \in E} \operatorname{inc}(o, e) .
$$

In the offline version of this problem, we will prove below that $T\left(o, e_{o p t}\right)$ is the optimal tour.

Lemma 1: For the offline exploration problem in a polygon $P$, the tour length of an offline optimal algorithm satisfies the following.

$$
|\mathrm{OPT}(P)|=L+\operatorname{inc}\left(o, e_{o p t}\right) .
$$

Proof : Let a permutation $\pi:\{1, \ldots, n\} \rightarrow\{1, \ldots, n\}$ denote the sequence of visiting polygon vertices for the searcher. Namely the searcher visits polygon vertices in the order of $v_{\pi(1)}, v_{\pi(2)}, \ldots, v_{\pi(n)}$ (see Fig. 1). Let $T_{\pi}$ denote the tour composed of paths $s p\left(o, v_{\pi(1)}\right) \rightarrow s p\left(v_{\pi(1)}, v_{\pi(2)}\right) \rightarrow$ $\cdots \rightarrow s p\left(v_{\pi(n)}, o\right)$, and $\left|T_{\pi}\right|$ be its length. Note that each polygon vertex must be visited in accordance with the order given by only $\pi$ even if it may happen that $T_{\pi}$ passes $v_{i}$ earlier

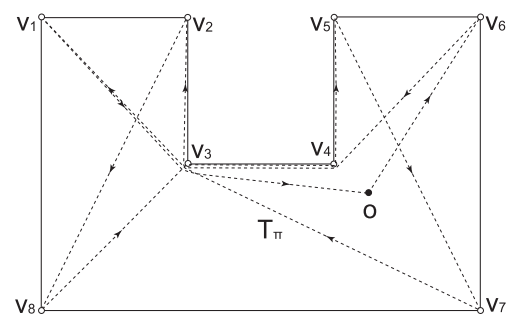

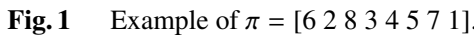

than specified by $\pi$ when $v_{i}$ is contained in $s p\left(v_{\pi(h)}, v_{\pi(h+1)}\right)$ for some $h<\pi^{-1}(i)$. In this case, even if $T_{\pi}$ passes through $v_{i}$ in $\operatorname{sp}\left(v_{\pi(h)}, v_{\pi(h+1)}\right)$, we consider $v_{i}$ is not visited by this part of $T_{\pi}$. If we show $\left|T_{\pi}\right| \geq\left|T\left(o, e_{o p t}\right)\right|$ for any $\pi$, then $|\mathrm{OPT}(P)| \geq\left|T\left(o, e_{\text {opt }}\right)\right|$ is shown. Furthermore, since $|\mathrm{OPT}(P)| \leq\left|T\left(o, e_{o p t}\right)\right|$ clearly holds, the lemma is proved.

At first we define an undirected graph $G=\left(V^{\prime}, E^{\prime}\right)$ from $T_{\pi}$ as follows. Let $V^{\prime}$ be composed of polygon vertices, self-intersection points of $T_{\pi}$ and the origin $o$. Also let $E^{\prime}$ be composed of line segments between consecutive vertices in $V^{\prime}$ along $T_{\pi}$. However we must make parallel edges where the searcher traverses an edge more than once. Note that $G$ is Eulerian. Let $E_{1}^{\prime}$ denote a set of outermost edges of $G$ (see Fig. 2) and $G_{1}=\left(V\left(E_{1}^{\prime}\right), E_{1}^{\prime}\right)$. Then $V\left(E_{1}^{\prime}\right)$ contains $V$ and $G_{1}$ is clearly Eulerian. There are two cases depending on a position of $o$.

Case 1: $o \in V\left(E_{1}^{\prime}\right)$. We regard $G_{1}$ as the clockwise tour from $o$, and without loss of generality we can assume that for some adjacent polygon vertices, say $v_{i}, v_{i+1} \in V$ with $1 \leq i \leq n, o$ is on the path from $v_{i}$ to $v_{i+1}$ on $G_{1}$. Note that the length of the shortest path from $o$ to $v_{i+1}$ on $G_{1}$ is at least $\left|s p\left(o, v_{i+1}\right)\right|$, the length of the clockwise path from $v_{i+1}$ to $v_{i}$ on $G_{1}$ is at least $\left|b p\left(v_{i+1}, v_{i}\right)\right|$ (since this path on $G_{1}$ visits clockwise all polygon vertices and $b p\left(v_{i+1}, v_{i}\right)$ is the shortest path from $v_{i+1}$ to $v_{i}$ which visits clockwise all polygon vertices), and the length of the shortest path from $v_{i}$ to $o$ on $G_{1}$ is at least $\left|s p\left(v_{i}, o\right)\right|$. Thus,

$$
\begin{aligned}
\left|T_{\pi}\right| & \geq\left|G_{1}\right| \geq\left|\operatorname{sp}\left(o, v_{i+1}\right)\right|+\left|b p\left(v_{i+1}, v_{i}\right)\right|+\left|\operatorname{sp}\left(v_{i}, o\right)\right| \\
& =\left|T\left(o, e_{i}\right)\right| \geq\left|T\left(o, e_{o p t}\right)\right| .
\end{aligned}
$$

Case 2: $o \notin V\left(E_{1}^{\prime}\right)$. Let $E_{2}^{\prime}=E \backslash E_{1}^{\prime}$ and $G_{2}=\left(V\left(E_{2}^{\prime}\right), E_{2}^{\prime}\right)$, and then let $G_{3}$ denote the connected component of $G_{2}$ which contains $o$. Let $u \in V^{\prime}$ be an intersection point of $G_{1}$ and $G_{3}$, and we assume that $u$ is on the path from $v_{i}$ to $v_{i+1}$ in $G_{1}$ for some $v_{i}, v_{i+1} \in V$ with $1 \leq i \leq n$. Clearly $G_{3}$ is also Eulerian, hence there are paths on $G_{3}, p_{1}$ from $o$ to $u$ and $p_{2}$ from $u$ to $o$, which share no edge. In the same way as Case 1 , we obtain $\left|G_{1}\right| \geq\left|s p\left(u, v_{i+1}\right)\right|+\left|b p\left(v_{i+1}, v_{i}\right)\right|+\left|s p\left(v_{i}, u\right)\right|$. Thus,

$$
\begin{aligned}
\left|T_{\pi}\right| & \geq\left|p_{1}\right|+\left|G_{1}\right|+\left|p_{2}\right| \\
& \geq|\operatorname{ss} p(o, u)|+\left|\operatorname{sp}\left(u, v_{i+1}\right)\right|+\left|b p\left(v_{i+1}, v_{i}\right)\right|+\left|s p\left(v_{i}, u\right)\right| \\
& \geq\left|T\left(o, e_{i}\right)\right| \geq\left|T\left(o, e_{o p t}\right)\right| .
\end{aligned}
$$

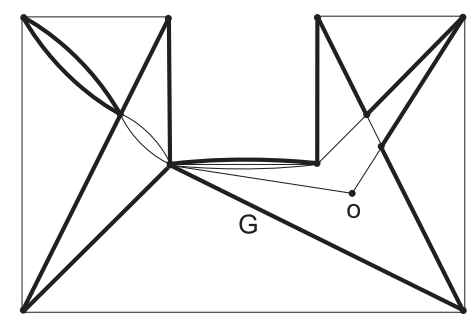

Fig. 2 Illustration of outermost edges of $G$ (represented by thick lines). 


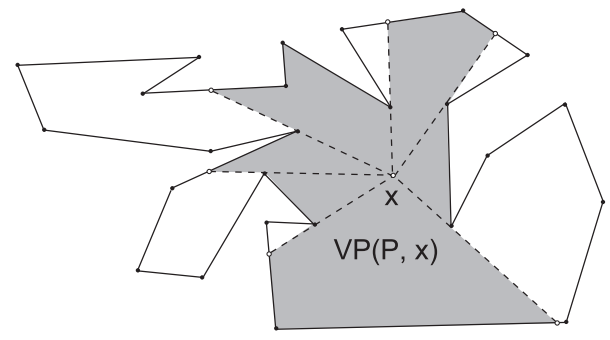

Fig. 3 Illustration of $V P(P, x)$ (represented by the shaded area).

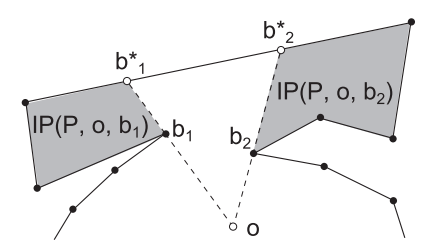

Fig. 4 Illustration of blocking vertices $b_{1}$ and $b_{2}$, virtual vertices $b_{1}^{*}$ and $b_{2}^{*}$, cut edges $b_{1} b_{1}^{*}$ and $b_{2} b_{2}^{*}$, virtual edge $b_{1}^{*} b_{2}^{*}$, and invisible polygons $I P\left(P, o, b_{1}\right)$ and $I P\left(P, o, b_{2}\right)$.

For two points $x, y \in P$, we say that $y$ is visible from $x$ if the line segment $x y$ contains no points of the outside of $P$. Then the visibility polygon $\operatorname{VP}(P, x)$ is

$$
V P(P, x):=\{y \in P \mid y \text { is visible from } x\} .
$$

Note that an edge of the visibility polygon is not necessarily a polygon edge (see Fig. 3). For a polygon vertex $b$ and a point $x \in P$, we call $b$ a blocking vertex with respect to $x$ if $b$ is visible from $x$ and there is the unique polygon edge incident to $b$ such that any point on the edge except $b$ is not visible from $x$. Let $b^{*}$ be a point where the extension of the line segment $x b$ towards $b$ first intersects the boundary of $P$. Then we call $b^{*}$ a virtual vertex and the line segment $b b^{*}$ a cut edge. Note that although a blocking vertex is always a polygon vertex, a virtual vertex may not coincide with any polygon vertex. Also let $\hat{e}$ be a polygon edge containing some virtual vertices then we regard a visible part of $\hat{e}$ as a new edge, which we call a virtual edge. Note that a cut edge $b b^{*}$ divides $P$ in two areas, a polygon which contains $V P(P, x)$ and the other not. We call the latter area an invisible polygon $\operatorname{IP}(P, x, b)$ (see Fig. 4). Notice that $V P(P, x)$ and $I P(P, x, b)$ share a cut edge $b b^{*}$. We assume that there is a blocking vertex $b$ with respect to the origin $o$ since otherwise an optimal solution can be found by Lemma 1. Then we have the following lemma.

Lemma 2: For an invisible polygon $\operatorname{IP}(P, o, b)$ defined by a blocking vertex $b$, let $e \in E$ be a polygon edge such that both of its endpoints are in $I P(P, o, b)$, and $w \in V$ be a polygon vertex adjacent to $b$ which is not in $I P(P, o, b)$. Then

$$
\operatorname{inc}(o,(b, w))<\operatorname{inc}(o, e) \text {. }
$$

Proof : First, we remark a simple fact. Let $x, y, z$ be points in $P$ such that both line segments $x z$ and $z y$ are lying in the inside of $P$. Then the following inequality obviously holds.

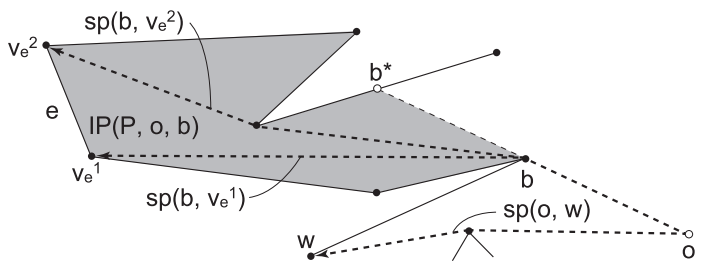

Fig. 5 Illustration of $s p\left(b, v_{e}^{1}\right), s p\left(b, v_{e}^{2}\right)$ and $s p(o, w)$ (the shaded area represents $I P(P, o, b))$.

$$
|\operatorname{sp}(x, y)| \leq|x z|+|z y| .
$$

Notice that the equality holds only when either (i) $s p(x, y)$ is a line segment $x y$ and $z$ is on $x y$, or (ii) $s p(x, y)$ is composed of two line segments $x z$ and $z y$, i.e., $y$ is not visible from $x$ and $z$ is a blocking vertex with respect to $x$.

See Fig. 5. From the above observation and since $b$ is visible from $o$, i.e., $|\operatorname{sp}(o, b)|=|o b|$,

$$
|s p(o, w)|<|o b|+|b w|=|s p(o, b)|+|b w| .
$$

Besides, from the triangle inequality with respect to $b, v_{e}^{1}$ and $v_{e}^{2}$,

$$
\operatorname{inc}(b, e)=\left|\operatorname{sp}\left(b, v_{e}^{1}\right)\right|+\left|s p\left(b, v_{e}^{2}\right)\right|-|e| \geq 0 .
$$

Furthermore both $s p\left(o, v_{e}^{1}\right)$ and $s p\left(o, v_{e}^{2}\right)$ pass through $b$. Hence, we have

$$
\begin{aligned}
|s p(o, b)|+\left|\operatorname{sp}\left(b, v_{e}^{1}\right)\right| & =\left|\operatorname{sp}\left(o, v_{e}^{1}\right)\right| \\
\text { and }|\operatorname{sp}(o, b)|+\left|\operatorname{sp}\left(b, v_{e}^{2}\right)\right| & =\left|\operatorname{sp}\left(o, v_{e}^{2}\right)\right| .
\end{aligned}
$$

Thus,

$$
\begin{aligned}
& \operatorname{inc}(o,(b, w))=|\operatorname{sp}(o, b)|+|\operatorname{sp}(o, w)|-|b w| \\
& <|\operatorname{ssp}(o, b)|+|\operatorname{spp}(o, b)|+|b w|-|b w| \\
& \leq 2|\operatorname{sp}(o, b)|+\left|\operatorname{sp}\left(b, v_{e}^{1}\right)\right|+\left|\operatorname{sp}\left(b, v_{e}^{2}\right)\right|-|e| \\
& =\operatorname{inc}(o, e)
\end{aligned}
$$

holds.

For $e_{\text {opt }}$ defined by (2), the following corollary is immediate from Lemma 2.

Corollary 1: For an invisible polygon $\operatorname{IP}(P, o, b)$ defined by a blocking vertex $b$, let $e \in E$ be a polygon edge both endpoints of which are in $\operatorname{IP}(P, o, b)$. Then $e$ cannot be $e_{o p t}$.

Based on Corollary 1, candidates of $e_{o p t}$ are polygon edges or virtual edges in $\operatorname{VP}(P, o)$.

In what follows, we propose an online algorithm, AOE(Avoiding One Edge). By Lemma 1, an offline optimal algorithm chooses a polygon edge $e_{\text {opt }}$ which satisfies (2). But we cannot obtain the whole information about $P$. So, the seemingly best strategy based on the information of $V P(P, o)$ is to choose an edge of $V P(P, o)$ in the same way as an offline optimal algorithm, assuming that there is no invisible polygon, namely $P=V P(P, o)$. Let $E_{1}^{*}$ denote a 
polygon edge set composed of all $e \in E$ such that both endpoints of $e$ are visible from $o, E_{2}^{*}$ denote a set of virtual edges on the boundary of $\operatorname{VP}(P, o)$ and $E^{*}=E_{1}^{*} \cup E_{2}^{*}$. Also for a virtual edge $e \in E_{2}^{*}$, endpoints of $e$ are labeled as $v_{e}^{1}, v_{e}^{2}$ in clockwise order around $o$ and as in (1), let inc $(o, e)$ denote the value of $\left|s p\left(o, v_{e}^{1}\right)\right|+\left|s p\left(o, v_{e}^{2}\right)\right|-|e|$. Let $e^{*} \in E^{*}$ be an edge satisfying the following equation.

$$
\operatorname{inc}\left(o, e^{*}\right)=\min _{e \in E^{*}} \operatorname{inc}(o, e)
$$

Then Algorithm AOE is described as follows.

Step 1: Choose $e^{*} \in E^{*}$ satisfying (7).

Step 2: If $e^{*} \in E_{1}^{*}$ then let $\hat{e}=e^{*}$, else let $\hat{e}$ be a polygon edge containing $e^{*}$.

Step 3: Follow the tour $T(o, \hat{e})$.

\section{Competitive Analysis of $\mathrm{AOE}$}

\subsection{Upper Bound for AOE}

First, we show the following lemma.

Lemma 3: Let $x$ be a point on the boundary of $P$ and $e^{*}$ be an edge satisfying (7). If $x$ is visible from the origin $o$, then

$$
\frac{\operatorname{inc}\left(o, e^{*}\right)}{2} \leq|o x| \text {. }
$$

Proof : Let $e^{\prime} \in E^{*}$ be an edge of $V P(P, o)$ containing $x$. Then from (3), we have $|o x| \geq\left|\operatorname{sp}\left(o, v_{e^{\prime}}^{1}\right)\right|-\left|x v_{e^{\prime}}^{1}\right|$ and $|o x| \geq$ $\left|s p\left(o, v_{e^{\prime}}^{2}\right)\right|-\left|x v_{e^{\prime}}^{2}\right|$. Therefore, we obtain

$$
\begin{aligned}
2|o x| & \geq\left|\operatorname{sp}\left(o, v_{e^{\prime}}^{1}\right)\right|+\left|\operatorname{sp}\left(o, v_{e^{\prime}}^{2}\right)\right|-\left|x v_{e^{\prime}}^{1}\right|-\left|x v_{e^{\prime}}^{2}\right| \\
& =\left|\operatorname{sp}\left(o, v_{e^{\prime}}^{1}\right)\right|+\left|\operatorname{sp}\left(o, v_{e^{\prime}}^{2}\right)\right|-\left|e^{\prime}\right| \geq \operatorname{inc}\left(o, e^{*}\right),
\end{aligned}
$$

namely $|o x| \geq \operatorname{inc}\left(o, e^{*}\right) / 2$.

Furthermore, we show a lemma which plays a crucial role in our analysis.

Lemma 4: Let $L$ be the length of the boundary of $P$ and $e^{*}$ be an edge satisfying (7). Then the following inequality holds.

$$
L \geq \pi \cdot \operatorname{inc}\left(o, e^{*}\right) .
$$

Proof : Let $C$ be a circle centered at the origin $o$ with the radius of $\operatorname{inc}\left(o, e^{*}\right) / 2$. From Lemma 3, any polygon edge does not intersect $C$. Thus $L$ is greater than the length of the circumference of $C$, namely

$$
L \geq 2 \pi \cdot \frac{\operatorname{inc}\left(o, e^{*}\right)}{2}=\pi \cdot \operatorname{inc}\left(o, e^{*}\right)
$$

holds.

Theorem 1: The competitive ratio of Algorithm AOE is at most 1.319 .
Proof : The tour length of Algorithm AOE obviously satisfies

$$
|\operatorname{AOE}(P)|=L+\operatorname{inc}\left(o, e^{*}\right) .
$$

On the other hand, the tour length of an offline optimal algorithm satisfies $|\mathrm{OPT}(P)|=L+i n c\left(o, e_{\text {opt }}\right)$ holds from Lemma 1. By the triangle inequality, inc $\left(o, e_{\text {opt }}\right) \geq 0$, namely $|\mathrm{OPT}(P)| \geq L$ holds. Thus we have

$$
\frac{|\operatorname{AOE}(P)|}{|\mathrm{OPT}(P)|} \leq \frac{L+\operatorname{inc}\left(o, e^{*}\right)}{L}=1+\frac{\operatorname{inc}\left(o, e^{*}\right)}{L} .
$$

From this and (8),

$$
\frac{|\operatorname{AOE}(P)|}{|\mathrm{OPT}(P)|} \leq 1+\frac{\operatorname{inc}\left(o, e^{*}\right)}{\pi \cdot \operatorname{inc}\left(o, e^{*}\right)}=1+\frac{1}{\pi} \leq 1.319
$$

is obtained.

Theorem 1 gives an upper bound of the competitive ratio. In the followings, we will obtain a better bound by a detailed analysis. First, we improve a lower bound of $|\mathrm{OPT}(P)|$. Note that for some points $x, y, z \in P$ such that both $y$ and $z$ are visible from $x$ and the line segment $y z$ is lying in the inside of $P$, we call $\angle y x z$ the visual angle at $x$ formed by $y z$.

Lemma 5: For an edge $e^{*} \in E^{*}$ satisfying (7), let $d=$ inc $\left(o, e^{*}\right)$ and $\theta(0 \leq \theta \leq \pi)$ be a visual angle at $o$ formed by a visible part of $e_{\text {opt }}$. Then

$$
|\mathrm{OPT}(P)| \geq L+d-d \sin \frac{\theta}{2} .
$$

Proof : We first show the following claim.

Claim 1: Let $b_{1} \in V$ (resp. $b_{2}$ ) be the polygon vertex visible from $o$ such that the path $\operatorname{sp}\left(o, v_{e_{o p t}}^{1}\right)\left(\operatorname{resp} . \operatorname{sp}\left(o, v_{e_{o p t}}^{2}\right)\right)$ passes through $b_{1}$ (resp. $b_{2}$ ) (see Fig. 6). Then

$$
\operatorname{inc}\left(o, e_{o p t}\right) \geq\left|o b_{1}\right|+\left|o b_{2}\right|-\left|b_{1} b_{2}\right| \text {. }
$$

Proof : This claim is obtained from $\left|s p\left(o, v_{e_{o p t}}^{1}\right)\right|=\left|o b_{1}\right|+$ $\left|s p\left(b_{1}, v_{e_{o p t}}^{1}\right)\right|,\left|s p\left(o, v_{e_{o p t}}^{2}\right)\right|=\left|o b_{2}\right|+\left|s p\left(b_{2}, v_{e_{o p t}}^{2}\right)\right|$ and $\left|e_{o p t}\right|=$ $\left|\operatorname{sp}\left(v_{e_{\text {opt }}}^{1}, v_{e_{\text {opt }}}^{2}\right)\right| \leq\left|\operatorname{sp}\left(b_{1}, v_{e_{\text {opt }}}^{1}\right)\right|+\left|b_{1} b_{2}\right|+\left|\operatorname{sp}\left(b_{2}, v_{e_{\text {opt }}}^{2}\right)\right|$.

From (10), we have

$$
|\mathrm{OPT}(P)|=L+\operatorname{inc}\left(o, e_{o p t}\right) \geq L+\left|o b_{1}\right|+\left|o b_{2}\right|-\left|b_{1} b_{2}\right| .
$$

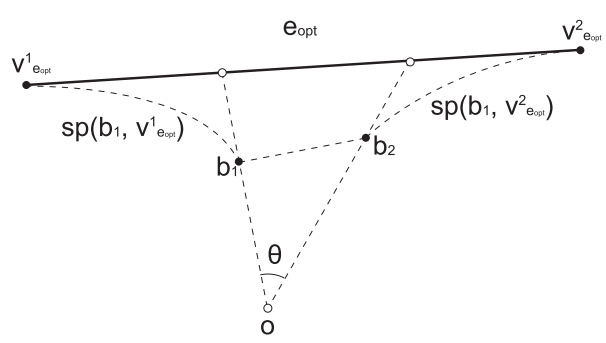

Fig. 6 Illustration of a visible part of $e_{\text {opt }}$ from $o$. 


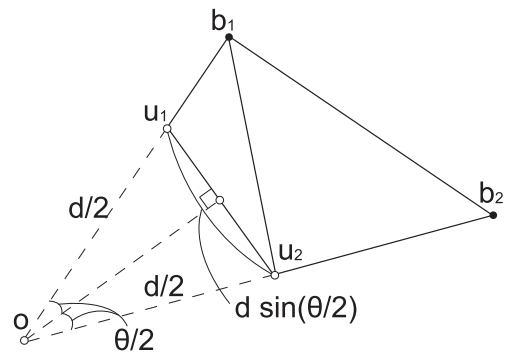

Fig. 7 Illustration of $u_{1}$ and $u_{2}$.

Furthermore $b_{1}$ and $b_{2}$ satisfy $\left|o b_{1}\right| \geq d / 2$ and $\left|o b_{2}\right| \geq d / 2$ from Lemma 3. Hence there exist points $u_{1}, u_{2}$ on line segments $o b_{1}, o b_{2}$ such that $\left|o u_{1}\right|=\left|o u_{2}\right|=d / 2$ (see Fig. 7). Then, from the triangle inequality with respect to $u_{1}, u_{2}$ and $b_{1}$,

$$
\left|u_{1} u_{2}\right| \geq\left|u_{2} b_{1}\right|-\left|b_{1} u_{1}\right|=\left|u_{2} b_{1}\right|-\left(\left|o b_{1}\right|-\frac{d}{2}\right)
$$

holds. Similarly we have

$$
\left|u_{2} b_{1}\right| \geq\left|b_{1} b_{2}\right|-\left|u_{2} b_{2}\right|=\left|b_{1} b_{2}\right|-\left(\left|o b_{2}\right|-\frac{d}{2}\right) .
$$

Thus we have

$$
\begin{aligned}
d-\left|u_{1} u_{2}\right| & \leq d-\left\{\left|u_{2} b_{1}\right|-\left(\left|o b_{1}\right|-\frac{d}{2}\right)\right\} \\
& \leq \frac{d}{2}+\left|o b_{1}\right|-\left\{\left|b_{1} b_{2}\right|-\left(\left|o b_{2}\right|-\frac{d}{2}\right)\right\} \\
& =\left|o b_{1}\right|+\left|o b_{2}\right|-\left|b_{1} b_{2}\right| .
\end{aligned}
$$

In addition, the length of $u_{1} u_{2}$ satisfies the following equation.

$$
\left|u_{1} u_{2}\right|=\frac{d}{2} \cdot 2 \sin \frac{\theta}{2}=d \sin \frac{\theta}{2} .
$$

By (11), (12) and (13),

$$
|\mathrm{OPT}(P)| \geq L+d-\left|u_{1} u_{2}\right|=L+d-d \sin \frac{\theta}{2}
$$

is shown.

Secondly, we show a better lower bound of $L$.

Lemma 6: Let $d$ and $\theta$ as defined in Lemma 5. Then

$$
L \geq d\left(\pi-\frac{\theta}{2}+\tan \frac{\theta}{2}\right)
$$

Proof : Let $C$ be a circle centered at $o$ with radius $d / 2$. From Lemma 3, any polygon edge does not intersect $C$. Also let endpoints of a visible part of $e_{o p t}$ from $o$ be $w_{1}, w_{2}$ in clockwise order around $o$. Then, we consider two cases; (Case 1) $\angle o w_{1} w_{2} \leq \pi / 2$ and $\angle o w_{2} w_{1} \leq \pi / 2$ and (Case 2) $\angle o w_{1} w_{2}>\pi / 2$ and $\angle o w_{2} w_{1} \leq \pi / 2$ (see Figs. 8, 9). Note that the case of $\angle o w_{1} w_{2} \leq \pi / 2, \angle o w_{2} w_{1}>\pi / 2$ can be treated in a manner similar to Case 2.

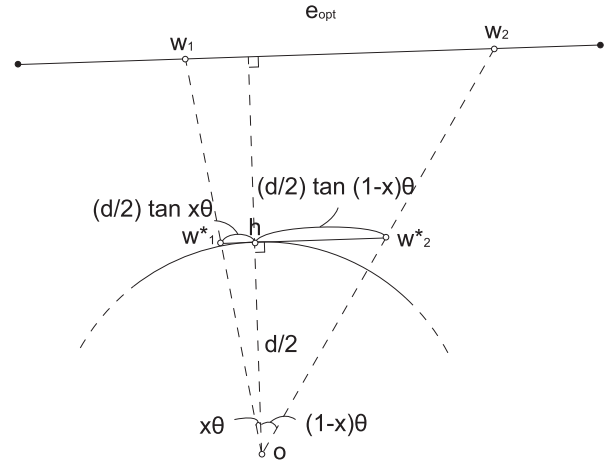

Fig. 8 Illustration of Case 1 in the proof of Lemma 6.

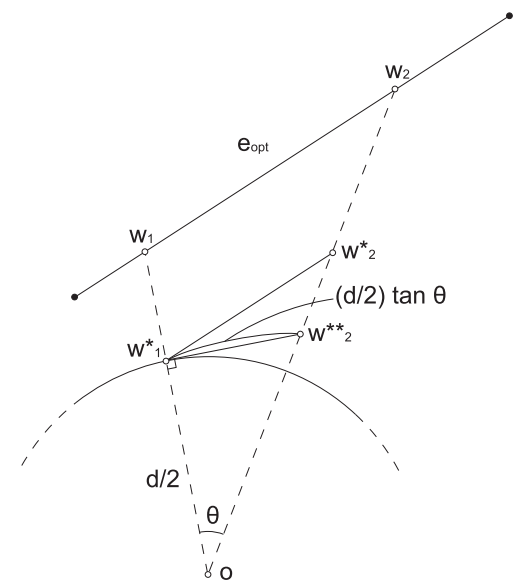

Fig. 9 Illustration of Case 2 in the proof of Lemma 6.

Case 1: Let $w_{1}^{*}$ (resp. $w_{2}^{*}$ ) be a point on the line segment $o w_{1}$ (resp. ow $w_{2}$ ) such that $w_{1} w_{2}$ is parallel to $w_{1}^{*} w_{2}^{*}$ and the line segment $w_{1}^{*} w_{2}^{*}$ touches the circle $C$ and let $h$ be a tangent point of $w_{1}^{*} w_{2}^{*}$ and $C$. Also let $\angle w_{1} o h=x \theta$ and $\angle w_{2} o h=$ $(1-x) \theta$ with some $x(0 \leq x \leq 1)$. Then the length of $w_{1}^{*} w_{2}^{*}$ satisfies

$$
\left|w_{1}^{*} w_{2}^{*}\right|=\frac{d}{2} \tan x \theta+\frac{d}{2} \tan (1-x) \theta .
$$

The right-hand side of this equation attains the minimum value when $x=1 / 2$. Thus

$$
\left|w_{1}^{*} w_{2}^{*}\right| \geq \frac{d}{2} \tan \frac{\theta}{2}+\frac{d}{2} \tan \frac{\theta}{2}=d \tan \frac{\theta}{2} .
$$

Furthermore the sum of the visual angle at $o$ formed by a visible part of the boundary other than $w_{1} w_{2}$ is equal to $2 \pi-$ $\theta$. Hence we have

$$
L \geq \frac{d}{2}(2 \pi-\theta)+\left|w_{1} w_{2}\right| .
$$

Since $\left|w_{1} w_{2}\right| \geq\left|w_{1}^{*} w_{2}^{*}\right|$ obviously holds, from (15) and (16), we obtain

$$
L \geq \frac{d}{2}(2 \pi-\theta)+d \tan \frac{\theta}{2}=d\left(\pi-\frac{\theta}{2}+\tan \frac{\theta}{2}\right) .
$$

Case 2: Let $w_{1}^{*}$ (resp. $w_{2}^{*}$ ) be a point on the line segment $o w_{1}$ 
(resp. ow $w_{2}$ such that $w_{1} w_{2}$ is parallel to $w_{1}^{*} w_{2}^{*}$ and $\left|o w_{1}^{*}\right|=$ $d / 2$ (the circumference of $C$ passes through $w_{1}^{*}$ ). Also let $w_{2}^{* *}$ an intersection point of the line segment $o w_{2}$ and the line perpendicular to the line segment $o w_{1}$ through $w_{1}^{*}$. Then

$$
\left|w_{1}^{*} w_{2}^{*}\right|>\left|w_{1}^{*} w_{2}^{* *}\right|=\frac{d}{2} \tan \theta \geq d \tan \frac{\theta}{2} .
$$

In the same way as Case 1 , we obtain $L \geq d(\pi-\theta / 2+$ $\tan (\theta / 2))$.

By Lemma 5 and 6, we prove the following theorem.

Theorem 2: The competitive ratio of Algorithm AOE is at most 1.219 .

Proof: Let $d$ and $\theta$ as defined in Lemma 5. Since $|\operatorname{AOE}(P)|=L+d$ holds, from (9), (14), we have

$$
\begin{aligned}
\frac{|\operatorname{AOE}(P)|}{|\operatorname{OPT}(P)|} & \leq \frac{L+d}{L+d-d \sin \frac{\theta}{2}} \\
& \leq \frac{d\left(\pi-\frac{\theta}{2}+\tan \frac{\theta}{2}\right)+d}{d\left(\pi-\frac{\theta}{2}+\tan \frac{\theta}{2}\right)+d-d \sin \frac{\theta}{2}} \\
& =\frac{\pi-\frac{\theta}{2}+\tan \frac{\theta}{2}+1}{\pi-\frac{\theta}{2}+\tan \frac{\theta}{2}+1-\sin \frac{\theta}{2}} \quad(0 \leq \theta \leq \pi) .
\end{aligned}
$$

In the followings, we compute the maximum value of (17),

$$
\max _{0 \leq \theta \leq \pi}\left\{z(\theta)=\frac{\pi-\frac{\theta}{2}+\tan \frac{\theta}{2}+1}{\pi-\frac{\theta}{2}+\tan \frac{\theta}{2}+1-\sin \frac{\theta}{2}}\right\} .
$$

Generally the following fact about the fractional program is known [1], [11].

Fact 1: Let $X \subseteq \mathbb{R}^{n}, f: \mathbb{R}^{n} \rightarrow \mathbb{R}$ and $g: \mathbb{R}^{n} \rightarrow \mathbb{R}$. Let us consider the following fractional program formulated as

$$
\operatorname{maximize}\left\{h(x)=\frac{f(x)}{g(x)} \mid x \in X\right\},
$$

where $g(x)>0$ is assumed for any $x \in X$. Let $x^{*} \in$ $\operatorname{argmax}_{x \in X} h(x)$ denote an optimal solution of (19) and $\lambda^{*}=h\left(x^{*}\right)$ denote the optimal value. Furthermore, with a real parameter $\lambda$, let $h_{\lambda}(x)=f(x)-\lambda g(x)$ and $M(\lambda)=$ $\max _{x \in X} h_{\lambda}(x)$. Then $M(\lambda)$ is monotone decreasing for $\lambda$ and the followings hold.

(i) $M(\lambda)<0 \Leftrightarrow \lambda>\lambda^{*}$, (ii) $M(\lambda)=0 \Leftrightarrow \lambda=\lambda^{*}$, (iii) $M(\lambda)>0 \Leftrightarrow \lambda<\lambda^{*}$.

In the same way as Fact 1 , with a real parameter $\lambda$, we define $z_{\lambda}(\theta)$ and $M(\lambda)$ for $z(\theta)$ as follows.

$$
\begin{aligned}
z_{\lambda}(\theta)= & \pi-\frac{\theta}{2}+\tan \frac{\theta}{2}+1 \\
& \quad-\lambda\left(\pi-\frac{\theta}{2}+\tan \frac{\theta}{2}+1-\sin \frac{\theta}{2}\right)(0 \leq \theta \leq \pi), \\
M(\lambda)= & \max _{0 \leq \theta \leq \pi} z_{\lambda}(\theta) .
\end{aligned}
$$

From Fact 1 (ii), $\lambda^{*}$ satisfying $M\left(\lambda^{*}\right)=0$ is equal to (18), i.e., the maximum value of $z(\theta)$. Hence we only need to compute $\lambda^{*}$.

Finally, let $\theta_{\lambda}^{*} \in \operatorname{argmax}_{0 \leq \theta \leq \pi} z_{\lambda}(\theta)$, then we show $\theta_{\lambda}^{*}$ is unique. A derivative of $z_{\lambda}(\theta)$ is calculated as

$$
\frac{d z_{\lambda}}{d \theta}=-\frac{\lambda-1}{2} \tan ^{2} \frac{\theta}{2}+\frac{\lambda}{2} \cos \frac{\theta}{2} .
$$

This derivative is monotone decreasing in the interval $0 \leq$ $\theta \leq \pi$, therefore $z_{\lambda}(\theta)$ is concave in this interval, then $\theta_{\lambda}^{*}$ is unique. Indeed when $\lambda=1.219, \theta_{\lambda}^{*} \simeq 2.0706$ then $M(1.219) \simeq-0.0010<0$. Also when $\lambda=1.218$, $\theta_{\lambda}^{*} \simeq 2.0718$ then $M(1.218) \simeq 0.0029>0$. Thus we obtain $1.218<\lambda^{*}<1.219$.

\subsection{Lower Bound for AOE}

Theorem 3: The competitive ratio of Algorithm AOE is at least 1.040 .

Proof : We consider how Algorithm AOE works for a polygon $P_{b a d}$ illustrated in Fig. 10. We assume that the greater arc from $h$ to $c$ in clockwise order of a circle with radius 10.00 centered at $o$ in the figure is in fact a chain composed of sufficiently many small polygon edges of length $\epsilon$. For each small edge $s$ along the arc $h c, \operatorname{inc}(o, s)=20.00-\epsilon$ holds. The algorithm calculates the increase of a virtual edge $(e, f)$ as $\operatorname{inc}(o,(e, f)) \simeq 10.00+8.18+10.00+8.18-$ $16.36=20.00$. Comparing these two values, the algorithm chooses a polygon edge $(a, b)$ in the arc $h c$. Since $L \simeq 136.26$ holds, the tour length of Algorithm AOE for $P_{\text {bad }}$ satisfies

$$
\left|\operatorname{AOE}\left(P_{b a d}\right)\right| \simeq 136.26+20.00-\epsilon \geq 156.26-\epsilon .
$$

On the other hand, $(d, g)=e_{\text {opt }}$ because inc $(o,(d, g)) \simeq$ $13.89<20.00-\epsilon$ holds. Thus the tour length of an offline optimal algorithm for $P_{b a d}$ satisfies

$$
\left|\mathrm{OPT}\left(P_{b a d}\right)\right| \simeq 136.26+13.89 \leq 150.16
$$

From (20) and (21), we obtain

$$
\frac{\left|\operatorname{AOE}\left(P_{b a d}\right)\right|}{\left|\operatorname{OPT}\left(P_{b a d}\right)\right|} \geq \frac{156.26-\epsilon}{150.16} \geq 1.0406-\frac{\epsilon}{150.16} .
$$

By letting $\epsilon$ be sufficiently small, the theorem follows.

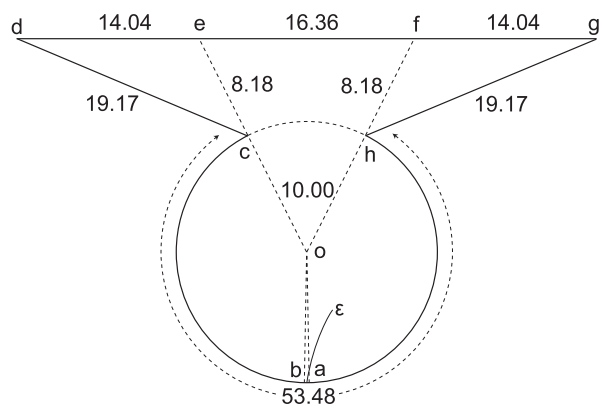

Fig. 10 Illustration of a polygon $P_{b a d}$ in the proof of Theorem 3. 


\section{Competitive Analysis for Rectilinear Polygon}

In this section, we analyze the competitive ratio of AOE for a rectilinear polygon (see Fig. 11). Generally a rectilinear polygon is defined as a simple polygon all of whose interior angles are $\pi / 2, \pi$ or $3 \pi / 2$. Polygon edges of the rectilinear polygon are classified as horizontal or vertical edges. Suppose that we are given a rectilinear polygon $R$ and the origin $o$ in $R$. Let $R^{\prime}$ be the minimum enclosing rectangle of $R$. Then we define the height of $R^{\prime}$ as the height of $R$ and also the width of $R^{\prime}$ as the width of $R$. Note that the searcher follows the Euclidean shortest path even if he/she is in the rectilinear polygon.

\subsection{Upper Bound for AOE}

Lemma 7: For an edge $e^{*} \in E^{*}$ satisfying (7), let $d=$ $\operatorname{inc}\left(o, e^{*}\right)$ and $\theta(0 \leq \theta \leq \pi)$ be a visual angle at $o$ formed by a visible part of $e_{\text {opt }}$. Then

$$
L \geq \max \left\{4 d, 2 d+2 d \tan \frac{\theta}{2}\right\} .
$$

Proof : First, we show $L \geq 4 d$. Let $C$ be a circle centered at $o$ with the radius of $d / 2$. From Lemma 3, any polygon edge of $R$ does not intersect $C$. Thus each of the height and width of $R$ is at least $d$ (the diameter of $C$ ), namely $L \geq 4 d$ holds (see Fig. 12).

Secondly, we show $L \geq 2 d+2 d \tan (\theta / 2)$. Note that we should just consider the case of $4 d \leq 2 d+2 d \tan (\theta / 2)$,

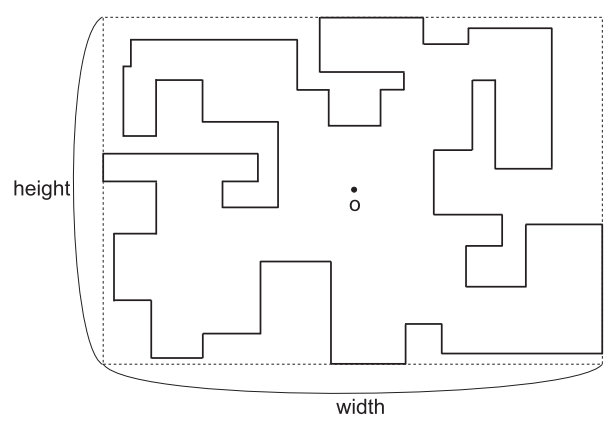

Fig. 11 Illustration of a rectilinear polygon.

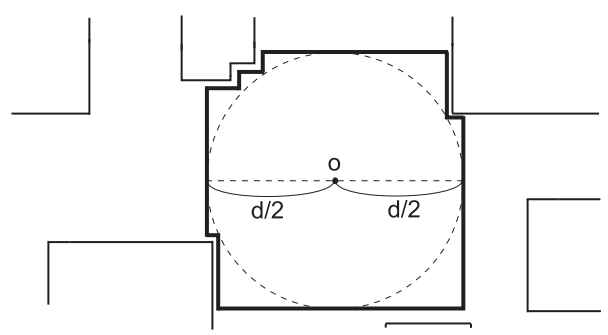

Fig. 12 Illustration of the minimum enclosing rectilinear polygon of $C$ (represented by thick lines) which is enclosed by $R$ (represented by thin lines). namely $\pi / 2 \leq \theta \leq \pi$ because $L \geq 4 d$ has been proved. Without loss of generality we can assume that $e_{\text {opt }}$ is a horizontal edge. We label endpoints of a visible part of $e_{o p t}$ from $o$ as $w_{1}, w_{2}$ in clockwise order around $o$. Let $w_{1}^{*}$ (resp. $\left.w_{2}^{*}\right)$ be a point on the line segment $o w_{1}$ (resp. $o w_{2}$ ) such that $w_{1} w_{2}$ is parallel to $w_{1}^{*} w_{2}^{*}$ and the line segment $w_{1}^{*} w_{2}^{*}$ touches the circle $C$ and $h$ be a tangent point of $w_{1}^{*} w_{2}^{*}$ and $C$ (see Fig. 13). Also let $\angle w_{1} o h=x \theta$ and $\angle w_{2} o h=(1-x) \theta$ with some $x(0 \leq x \leq 1)$. Then the length of $w_{1}^{*} w_{2}^{*}$ satisfies

$$
\begin{aligned}
\left|w_{1}^{*} w_{2}^{*}\right| & =\frac{d}{2} \tan x \theta+\frac{d}{2} \tan (1-x) \theta \\
& \geq \frac{d}{2} \tan \frac{\theta}{2}+\frac{d}{2} \tan \frac{\theta}{2}=d \tan \frac{\theta}{2} .
\end{aligned}
$$

Thus the width of $R$ is at least $d \tan (\theta / 2)$ and the height of $R$ is at least $d$, then $L \geq 2 d+2 d \tan (\theta / 2)$ holds.

Theorem 4: For a rectilinear polygon, the competitive ratio of Algorithm AOE is at most 1.167.

Proof : Based on (22), we consider two cases; (Case 1) $0 \leq \theta<\pi / 2$ and (Case 2) $\pi / 2 \leq \theta \leq \pi$. Note that $4 d>$ $2 d+2 d \tan (\theta / 2)$ holds in Case 1 and $4 d \leq 2 d+2 d \tan (\theta / 2)$ holds in the other.

Case 1: From $L \geq 4 d$ and (9), we obtain

$$
\begin{aligned}
\frac{|\mathrm{AOE}(P)|}{|\mathrm{OPT}(P)|} & \leq \frac{4 d+d}{4 d+d-d \sin \frac{\theta}{2}}=\frac{5}{5-\sin \frac{\theta}{2}} \\
& <\frac{5}{5-\sin \frac{\pi}{4}} \leq 1.165 .
\end{aligned}
$$

Case 2: From $L \geq 2 d+2 d \tan (\theta / 2)$ and (9), we obtain

$$
\begin{aligned}
\frac{|\mathrm{AOE}(P)|}{|\mathrm{OPT}(P)|} & \leq \frac{2 d+2 d \tan \frac{\theta}{2}+d}{2 d+2 d \tan \frac{\theta}{2}+d-d \sin \frac{\theta}{2}} \\
& =\frac{3+2 \tan \frac{\theta}{2}}{3+2 \tan \frac{\theta}{2}-\sin \frac{\theta}{2}} .
\end{aligned}
$$

We will compute the maximum value of (23) as in the proof of Theorem 2 by defining $z_{\lambda}(\theta)$ and $M(\lambda)$ for a real parameter $\lambda$ as follows.

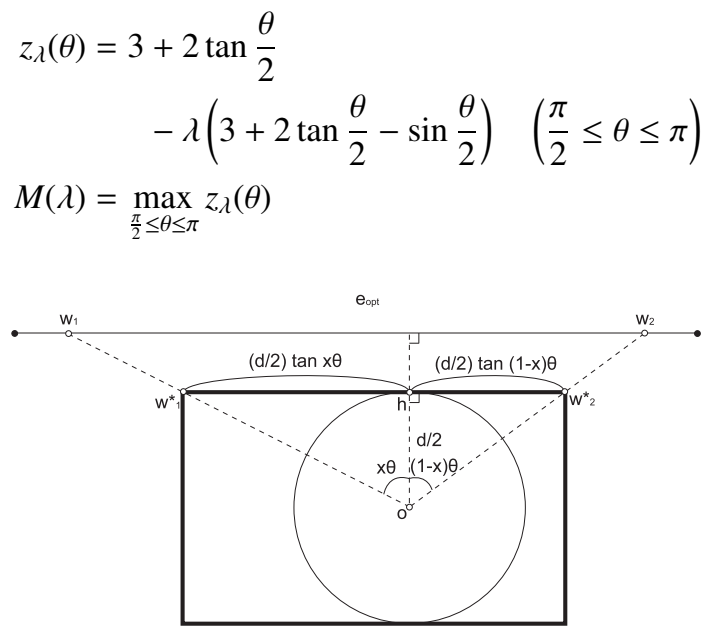

Fig. 13 Illustration of the minimum enclosing rectangle of $C$ (represented by thick lines) such that $\theta$ is more than $\pi / 2$. 
Let $\theta_{\lambda}^{*} \in \operatorname{argmax}_{0 \leq \theta \leq \pi} z_{\lambda}(\theta)$, then a derivative of $z_{\lambda}(\theta)$ is calculated as

$$
\frac{d z_{\lambda}}{d \theta}=-(\lambda-1) \frac{1}{\cos ^{2} \frac{\theta}{2}}+\frac{\lambda}{2} \cos \frac{\theta}{2} .
$$

This derivative is monotone decreasing in the interval $\pi / 2 \leq$ $\theta \leq \pi$, therefore $z_{\lambda}(\theta)$ is concave in this interval, then $\theta_{\lambda}^{*}$ is unique. Indeed when $\lambda=1.167, \theta_{\lambda}^{*} \simeq 1.7026$ then $M(1.167) \simeq-0.0044<0$. Also when $\lambda=1.166, \theta_{\lambda}^{*} \simeq$ 1.7056 then $M(1.166) \simeq 7.6 \times 10^{-5}>0$. Thus we obtain $1.166<\lambda^{*}<1.167$.

\subsection{Lower Bound for AOE}

Theorem 5: The competitive ratio of Algorithm AOE for a rectilinear polygon is at least 1.034 .

Proof : We consider how Algorithm AOE works for a polygon $R P_{b a d}$ illustrated in Fig. 14 . Let $\widetilde{m f}$ denote the polygonal chain from $m$ to $f$ in clockwise order around $o$ composed of segments $m n, n p, p q, q c, c d$, de and $e f$ in the figure. We assume that $\widetilde{m f}$ is in fact a chain composed of sufficiently many small polygon edges of length $\epsilon$. Notice that segments $m n$ and $e f$ are edges of length $\epsilon$. Also we can assume that $a b$ is a polygon edge in the middle of $q$ and $c$ such that $|o a|=|o b| \simeq 10.00$. Then $\operatorname{inc}(o,(a, b)) \simeq 20.00-\epsilon$ and $\operatorname{inc}(o, s) \geq 20.00-\epsilon$ holds for each small edge $s$ along $\widetilde{m f}$. The algorithm calculates the increase of a virtual edge $(i, j)$ as $\operatorname{inc}(o,(i, j)) \simeq 10.00+8.56+10.00+8.56-17.12=$ 20.00. Thus the algorithm chooses the polygon edge $(a, b)$. Since $L \simeq 172.48$ holds, the tour length of Algorithm AOE for $R P_{b a d}$ satisfies

$$
\left|\mathrm{AOE}\left(R P_{b a d}\right)\right| \simeq 172.48+20.00-\epsilon \geq 192.48-\epsilon .
$$

On the other hand, $(h, k)=e_{\text {opt }}$ because inc $(o,(h, k)) \simeq$ $13.58<20.00-\epsilon$ holds. Thus the tour length of an offline optimal algorithm for $R P_{b a d}$ satisfies

$$
\left|\mathrm{OPT}\left(R P_{\text {bad }}\right)\right| \simeq 172.48+13.58 \leq 186.07 \text {. }
$$

From (24) and (25), we obtain

$$
\frac{\left|\operatorname{AOE}\left(R P_{b a d}\right)\right|}{\left|\operatorname{OPT}\left(R P_{b a d}\right)\right|} \geq \frac{192.48-\epsilon}{186.07} \geq 1.0344-\frac{\epsilon}{186.07} \text {. }
$$

By letting $\epsilon$ be sufficiently small, the theorem follows.

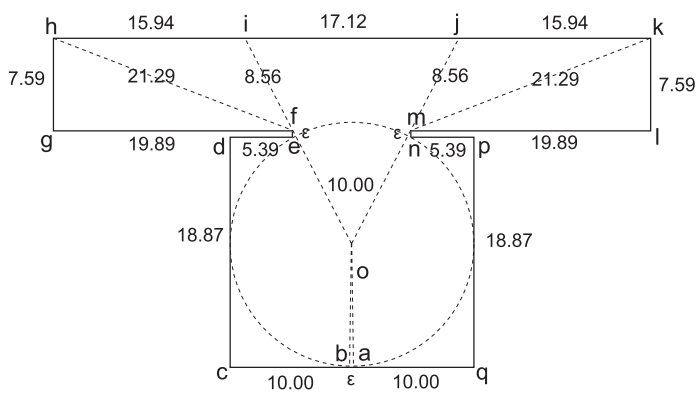

Fig. 14 Illustration of a rectilinear polygon $R P_{\text {bad }}$ in the proof of Theorem 5.

\section{Discussion and Open Problems}

In Lemma 5, the lower bound of $|\mathrm{OPT}(P)|$ given by (9) is not tight, and in Lemma 6, the lower bound of the boundary length $L$ given by (14) is also not tight. Hence, we believe that the upper bound of the competitive ratio can be improved: the least upper bound for a simple polygon (resp. a rectilinear polygon) could be close to the lower bound 1.040 (resp. 1.034) given in Sect. 3.2.

As one of many variations of OVEP, we could consider OVEP with multiple searchers. In this problem, all searchers are initially at the same origin $o \in P$. The goal of the exploration is that each polygon vertex is visited by at least one searcher and that all searchers return to the origin $o$. We regard the time when the last searcher comes back to the origin as the cost of the exploration. Note that our algorithm can be easily adapted to the case of OVEP with 2 -searchers. For an offline exploration problem with $k$-searchers, Frederickson et al. [4] proposed a $(e+1-1 / k)$-approximation algorithm, where $e$ is the approximation ratio of some 1-searcher algorithm. Their idea is splitting a tour given by some 1-searcher algorithm into $k$ parts such that the cost of each part is equal, where the cost of a part is the length of the shortest tour from $o$ which passes along the part. When $k=2$, we can apply this idea to our algorithm as follows. First, choose similarly $e^{*} \in E^{*}$ satisfying (7). Then let one searcher go to $v_{e^{*}}^{1}$ and walk counterclockwise along the boundary of $P$, and let symmetrically the other go to $v_{e^{*}}^{2}$ and walk clockwise. When two searchers meet at a point on the boundary, two searchers come back together to $o$ along the shortest path in the inside of $P$. In this case, we obtain an upper bound 1.719. However, when $k \geq 3$, the above-mentioned idea cannot be directly applied. So, it remains open.

\section{References}

[1] W. Dinkelbach, "On nonlinear fractional programming," Management Science, vol.13, no.7, pp.492-498, 1967.

[2] M. Dynia, J. Lopuszański, and C. Schindelhauer, "Why robots need maps," Proc. SIROCCO 2007, LNCS 4474, pp.41-50, 2007.

[3] P. Fraigniaud, L. Gsieniec, D.R. Kowalski, and A. Pelc, "Collective tree exploration," Networks, vol.48, no.3, pp.166-177, 2006.

[4] G.N. Frederickson, M.S. Hecht, and C.E. Kim, "Approximation algorithms for some routing problems," SIAM J. Comput., vol.7, pp.178-193, 1978.

[5] S.K. Ghosh and R. Klein, "Online algorithms for searching and exploration in the plane," Computer Science Review, vol.4, no.4, pp.189-201, 2010.

[6] Y. Higashikawa, N. Katoh, S. Langerman, and S. Tanigawa, "Online graph exploration algorithms for cycles and trees by multiple searchers," Proc. 3rd AAAC Annual Meeting, 2010.

[7] F. Hoffmann, C. Icking, R. Klein, and K. Kriegel, "The polygon exploration problem," SIAM J. Comput., vol.31, no.2, pp.577-600, 2002.

[8] N. Megow, K. Mehlhorn, and P. Schweitzer, "Online graph exploration: New results on old and new algorithms," Proc. 38th ICALP, LNCS 6756, pp.478-489, 2011.

[9] S. Miyazaki, N. Morimoto, and Y. Okabe, "The online graph exploration problem on restricted graphs," IEICE Trans. Inf. \& Syst., vol.E92-D, no.9, pp.1620-1627, Sept. 2009. 
[10] B. Kalyanasundaram and K.R. Pruhs, "Constructing competitive tours from local information," Theoretical Computer Science, vol.130, pp.125-138, 1994.

[11] S. Schaible and T. Ibaraki, "Fractional programming," European Journal of Operational Research, vol.12, pp.325-338, 1983.

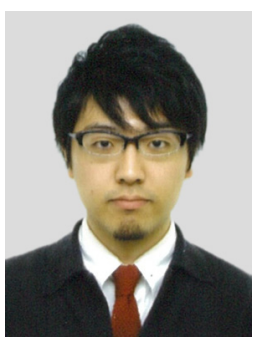

Yuya Higashikawa received the B.Eng. and M.Eng. degrees in Architecture and Architectural Engineering from Kyoto University in 2008 and 2010, respectively. He is currently a Ph.D. student at Department of Architecture and Architectural Engineering, Kyoto University. He is a member of IPSJ.

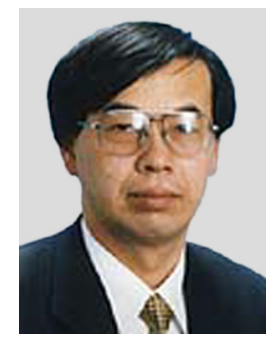

Naoki Katoh received the B.Eng., M.Eng. and Dr.Eng. degrees in Applied Mathematics and Physics from Kyoto University in 1973, 1975 and 1981, respectively. He was an Assistant Professor during 1981-1982, an Associate Professor during 1982-1990, and a Professor during 1990-1997, respectively, at Department of Management Science of Kobe University of Commerce. In 1997 he joined Kyoto University where he is currently a Professor at Department of Architecture and Architectural Engineering. His research interests include the design and analysis of combinatorial and geometric algorithms, data mining and architectural information systems. He is a member of IPSJ, OR Soc. Japan, Japan SIAM, and ACM. 